\title{
Analysis of anti-malarial resistance markers in pfmdr 1 and pfcrt across Southeast Asia in the Tracking Resistance to Artemisinin Collaboration
}

\author{
Krongkan Srimuang ${ }^{1}$, Olivo Miotto ${ }^{2,3,4}$, Pharath Lim ${ }^{5}$, Rick M. Fairhurst ${ }^{5}$, Dominic P. Kwiatkowski ${ }^{3,4}$, \\ Charles J. Woodrow ${ }^{2,6}$, Mallika Imwong ${ }^{1,2^{*}}$ and for the Tracking Resistance to Artemisinin Collaboration
}

\begin{abstract}
Background: Declining anti-malarial efficacy of artemisinin-based combination therapy, and reduced Plasmodium falciparum susceptibility to individual anti-malarials are being documented across an expanding area of Southeast Asia (SEA). Genotypic markers complement phenotypic studies in assessing the efficacy of individual anti-malarials.

Methods: The markers pfmdr1 and pfcrt were genotyped in parasite samples obtained in 2011-2014 at 14 TRAC (Tracking Resistance to Artemisinin Collaboration) sites in mainland Southeast Asia using a combination of PCR and next-generation sequencing methods.

Results: Pfmdr1 amplification, a marker of mefloquine and lumefantrine resistance, was highly prevalent at Mae Sot on the Thailand-Myanmar border (59.8\% of isolates) and common (more than 10\%) at sites in central Myanmar, eastern Thailand and western Cambodia; however, its prevalence was lower than previously documented in Pailin, western Cambodia. The pfmdr 1 Y 184F mutation was common, particularly in and around Cambodia, and the F1226Y mutation was found in about half of samples in Mae Sot. The functional significance of these two mutations remains unclear. Other previously documented pfmdr 1 mutations were absent or very rare in the region. The pfcrt mutation K76T associated with chloroquine resistance was found in $98.2 \%$ of isolates. The CVIET haplotype made up $95 \%$ or more of isolates in western SEA while the CVIDT haplotype was common (30-40\% of isolates) in north and northeastern Cambodia, southern Laos, and southern Vietnam.
\end{abstract}

Conclusions: These findings generate cause for concern regarding the mid-term efficacy of artemether-lumefantrine in Myanmar, while the absence of resistance-conferring pfmdr1 mutations and SVMNT pfcrt haplotypes suggests that amodiaquine could be an efficacious component of anti-malarial regimens in SEA.

Keywords: Malaria, Plasmodium falciparum, Anti-malarial resistance, Chloroquine, Amodiaquine, Mefloquine, Artemisinin, Combination therapy, pfcrt, pfmdr1

\section{Background}

Anti-malarial resistance in Plasmodium falciparum has originated and spread from Southeast Asia (SEA) on multiple occasions, and the high global prevalence of

\footnotetext{
*Correspondence: noi@tropmedres.ac

1 Department of Molecular Tropical Medicine and Genetics, Faculty

of Tropical Medicine, Mahidol University, Bangkok, Thailand

Full list of author information is available at the end of the article
}

chloroquine and antifolate resistance has made these drugs ineffective in the vast majority of malaria-endemic areas. In SEA, artemisinin-based combination therapies (ACTs) that combine mefloquine, lumefantrine, or piperaquine with an artemisinin derivative are currently used as front-line treatments. However, emerging resistance to artemisinins [1-5] and their partner drugs is causing ACT cure rates to fall below acceptable levels at an increasing number of sites in SEA [6-10]. When 
choosing the ideal ACT for a given location, validated molecular resistance markers can provide useful data that complement the results of clinical trials and in vitro studies, and potentially identify resistance trends at a relatively early stage [11].

The global spread of chloroquine resistance was primarily caused by mutant haplotypes in pfcrt, often accompanied by additional mutations in pfmdr1 (corresponding to codons 86, 184, 1034, 1042, and 1246). Paradoxically, some of these $p f m d r 1$ mutations are associated with hypersensitivity to mefloquine and lumefantrine [12, 13]; accordingly, switching to a drug regimen containing these latter drugs first leads to reselection of pfmdr1 wild-type alleles [14]. True resistance to mefloquine and lumefantrine then arises through amplification of pfmdr1 [15, 16], and is associated with clinical failure of the ACT artesunate-mefloquine $[15,17,18]$ and artemether-lumefantrine [14].

The prevalence of different molecular markers of resistance has been described in certain parts of SEA, but not in many other areas, notably Myanmar, the country with the highest malaria caseload. Here, relevant mutations in $p f c r t$ and $p f m d r 1$ were examined in samples from the Tracking Resistance to Artemisinin Collaboration (TRAC) study [5], covering 14 sites in six countries (Fig. 1).

\section{Methods}

\section{Sample collection}

Samples were collected as part of the TRAC study, a clinical trial focused on artemisinin resistance, between May 2011 and April 2013 [5]. Samples from three additional (TRAC Continuation) sites in Myanmar were collected in 2013-2014 (Fig. 1; Table 1).

Samples were analysed by PCR in the Molecular Malaria Laboratory, Faculty of Tropical Medicine, Bangkok, Thailand and by whole-genome sequencing (WGS) at the Wellcome Trust Sanger Institute, Hinxton, UK [19]. PCR was used to assess $p f m d r 1$ copy number and five well-described single-nucleotide polymorphisms (SNPs) in $p f m d r 1$ for approximately 30 samples from each site. WGS data covering all SNPs in $p f m d r 1$ and the classical pfcrt haplotypes encoding amino acids 72-76, were available for all samples tested. Because of timing and logistical issues, samples from the three additional sites in Myanmar were only analysed by PCR. Pfmdr1 copy number measurements for all 120 samples from each of three Cambodian sites (Pursat, Preah Vihear, Ratanakiri) have already been reported [20] and were included in the overall analysis (Table 1 ).

\section{DNA processing and whole-genome sequencing}

Admission blood samples were anticoagulated in EDTA, washed in PBS, and filtered through a cellulose CF11 column to deplete host leukocytes [21]. Genomic DNA was extracted using QIAamp ${ }^{\circledR}$ DNA Mini Kit (QIAGEN, Germany), following the manufacturer's instructions. Eluted genomic DNA samples were quantified by PicoGreen analysis and quantitative real-time PCR using the Applied Biosystems StepOne RT-PCR system and frozen at $-80^{\circ} \mathrm{C}$.

Samples with more than 50 ng DNA and less than 80\% human DNA contamination were submitted for WGS using the Illumina Genome Analyzer II platform. The procedure for sequencing, assembly of sequencing reads, variant calling, quality filtering, and genotype calling has been fully described elsewhere $[19,22]$. Each sample was genotyped at each of 926,988 high-quality exonic positions [19], not all of which were polymorphic within the sample set. Naturally this included the $p f c r t$ and $p f m d r 1$ resistance markers. Genome-wide genotype data were used to compute a genetic distance matrix from which a neighbour-joining tree was constructed, as previously described [19].

\section{Analysis of WGS read depth}

In each sample, the coverage depth $\left(c_{i}\right)$ at each position (i.e., the number of sequencing reads that cover that position in the sample alignment) was computed, from which the median coverage of the sample $\left(c_{m}\right)$ was determined. The relative coverage $\left(c_{r}\right)$ at each position was then obtained by $c_{r}=c_{i} c_{m}$. Since such a large proportion of the $P$. falciparum genome is very unevenly covered [22], $c_{r}$ was not directly used to estimate copy number. Rather, a number of reference positions were identified that exhibited consistent coverage across the complete sample set and were located in $p f m d r 1$ or genes with similar characteristics.

Seven reference positions were identified in two regions of $p f m d r 1$ that exhibited even coverage and GC content; their spacing ( $250 \mathrm{bp})$ is such that coverage is not affected by the same reads, and they have very low minor allele frequency (MAF) to minimize the probability of mis-mappings. For each sample, the relative coverage of $p$ fmdr1 $\left(c_{m d r 1}\right)$ was estimated as the median of $c_{r}$ at the $p f m d r 1$ reference positions.

A further 56 reference positions were identified in genes similar to $p f m d r 1$, i.e., with conservation score estimated as previously described [23] in the range 3.2-4.0 (pfmdr1 conservation score $=3.6$ ), and a single exon of size $>3 \mathrm{kbp}$. The positions were chosen within exonic regions $>300$ nucleotides devoid of high-frequency SNPs, with similar GC content ( $24 \%)$ and median coverage to $p f m d r 1$. Each reference position was chosen to have a limited coverage range variation across all samples in the MalariaGEN P. falciparum Community Project [24], with inter-quartile range (IQR) boundaries within $15 \%$ of 


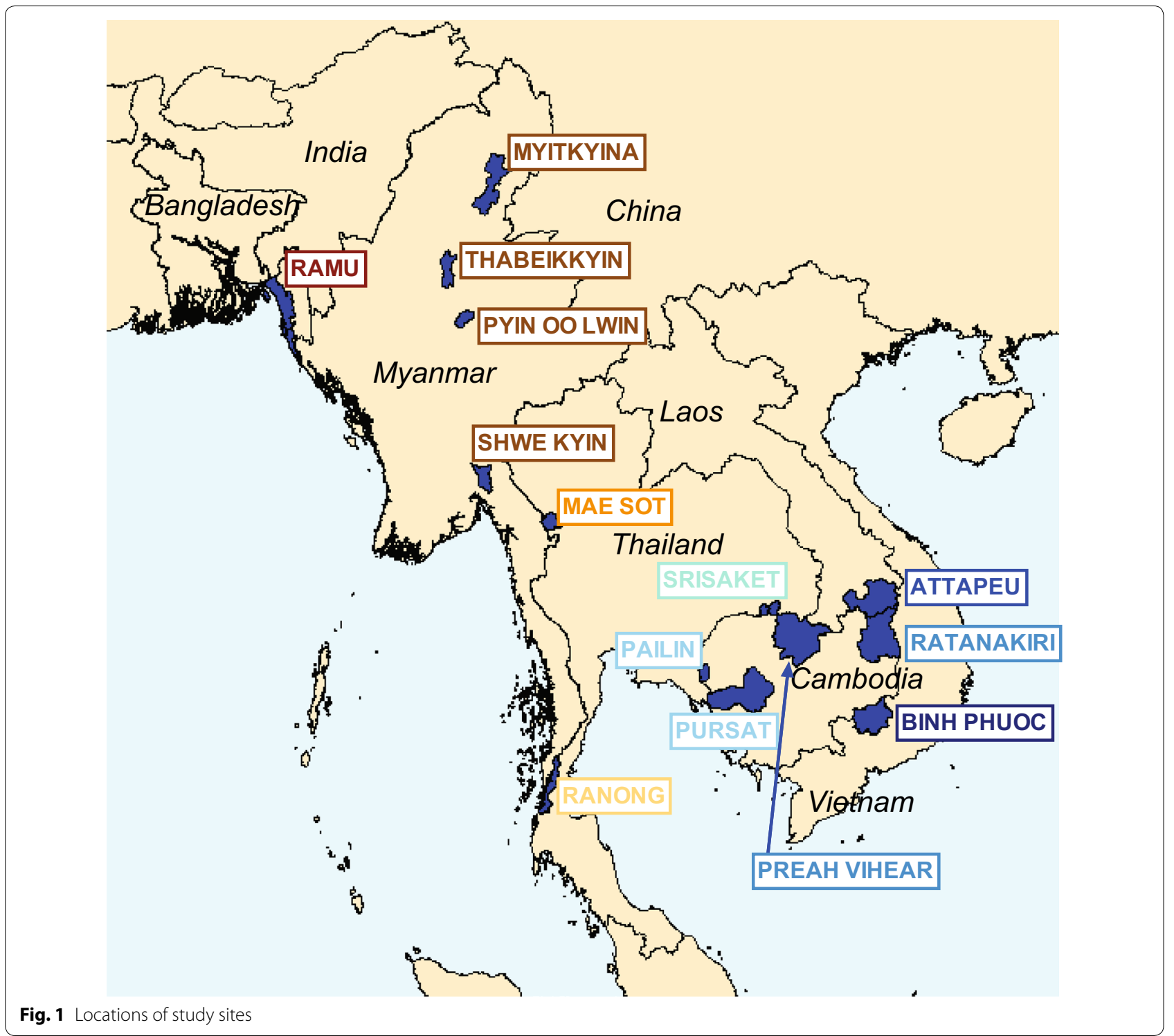

$c_{m d r 1}$. The selected reference positions are listed in Additional file 1 . Coverage statistics at these positions can be visualized using the $P$. falciparum Community Project web application [24]; see Additional file 2.

For each sample, the reference relative coverage $\left(c_{r e f}\right)$ was calculated as the median of $c_{r}$ at the 56 reference positions outside $p f m d r 1$. The $p f m d r 1$ copy number was thus estimated to be $N_{e s t}=c_{m d r 1} c_{r e f}$ A plot of the distribution of $N_{\text {est }}$ in the present sample set (Additional file 3) shows clear peaks at integer values. $P f m d r 1$ amplification was defined as copy number $>1.5$.

\section{Pfcrt haplotype determination}

Due to the high number of small exons, the low complexity of the introns, and the extreme levels of polymorphism around the key drug-resistance variation site, $p f c r t$ is a very difficult gene to assemble from Illumina short-read sequence data, even in otherwise well-covered samples. As a result, the previously used routine genotype calling method [22] is unable to determine pfcrt genotypes in many samples. To overcome this problem, a novel bespoke procedure was used for genotyping the core pfcrt haplotype (defined as amino acid positions 72-76).

Each flank of the core pfcrt haplotype contains a significant number of positions which are invariant in all the P. falciparum Community Project samples, forming two invariant flanking sequences: TATTATTTATTTAAGTGTA upstream of the core pfcrt haplotype and ATTTTTGCTAAAAGAAC downstream. The application samtools V1.2 [25] was used to extract all sequencing 
Table 1 Summary of Southeast Asian samples studied

\begin{tabular}{|c|c|c|c|c|c|c|c|}
\hline Year & Site & Province & Country & $\begin{array}{l}\text { Total samples } \\
\text { available }\end{array}$ & $\begin{array}{l}\text { Samples analysed } \\
\text { by PCR (Thailand) }\end{array}$ & $\begin{array}{l}\text { Samples analysed } \\
\text { by Illumina (UK) }\end{array}$ & $\begin{array}{l}\text { Samples analysed } \\
\text { by PCR (USA) }\end{array}$ \\
\hline \multicolumn{8}{|l|}{ TRAC study } \\
\hline 2012 & Ramu & Cox's Bazar & Bangladesh & 51 & 30 & 48 & \\
\hline $2011-2012$ & Pailin & Pailin & Cambodia & 90 & 33 & 84 & \\
\hline $2011-2012$ & Pursat & Pursat & Cambodia & 120 & & 98 & 120 \\
\hline 2011-2012 & Preah Vihear & Preah Vihear & Cambodia & 120 & & 96 & 120 \\
\hline 2011-2012 & Ratanakiri & Ratanakiri & Cambodia & 120 & & 93 & 120 \\
\hline 2011-2012 & Attapeu & Attapeu & Laos & 86 & 37 & 85 & \\
\hline 2012 & Shwe Kyin & Bago & Myanmar & 64 & 30 & 61 & \\
\hline $2011-2012$ & Mae Sot & Tak & Thailand & 107 & 38 & 104 & \\
\hline 2011-2013 & Srisaket & Srisaket & Thailand & 36 & 33 & 21 & \\
\hline $2011-2012$ & Kraburi & Ranong & Thailand & 23 & 23 & 20 & \\
\hline 2011-2012 & Bu Gia map & Binh Phuoc & Vietnam & 102 & 29 & 97 & \\
\hline \multicolumn{8}{|c|}{ TRAC continuation study } \\
\hline 2012-2014 & Pyin Oo Lwin & Mandalay & Myanmar & 29 & 29 & & \\
\hline 2013-2014 & Thabeikkyin & Mandalay & Myanmar & 30 & 30 & & \\
\hline \multirow[t]{2}{*}{2013} & Myitkyina & Kachin & Myanmar & 20 & 20 & & \\
\hline & & & Total & 998 & 332 & 807 & 360 \\
\hline
\end{tabular}

a Previously described [20]

reads containing the two flanking sequences (or their reverse complement) from each sample's alignment. The reads were then aligned against the $\mathrm{V} 33 \mathrm{D} 7$ pfcrt reference sequence (GeneDB PF3D7_0709000), after discarding low-quality reads (i.e., those carrying phred scores lower than 20 in the core haplotype codons). The core haplotype for each sample was finally read directly from the resulting alignment.

\section{PCR methods}

Pfmdr1 amplification undertaken in Thailand utilized Taqman real-time PCR (Rotor Gene 3000; Corbett Research, Australia) following established procedures and using published primers $[15,26]$. In each set of reactions, the 3D7 P. falciparum strain (single copy) was used as a calibrator (in triplicate). All reaction sets also included a previously derived positive control DNA extract with an estimated pfmdr1 copy number of 2.3 [27]; any run in which this gave a result of fewer than two or more than three copies was re-tested; in 27 separate runs the mean copy number was 2.34 (95\% CI 2.25-2.43, range $2.06-$ 2.83). A negative control (reagents only) was also tested each time. The threshold cycle $(\mathrm{Ct})$ of samples was calculated by the $\Delta \Delta \mathrm{Ct}$ calculation for the relative quantification of target: $\Delta \Delta \mathrm{Ct}=(\mathrm{Ct} p f m d r 1-\mathrm{Ct} p f \beta$-tubulin $)$ of sample - (Ct pfmdr1 - Ct pf $\beta$-tubulin) of P. falciparum 3D7. Copy number was calculated by the formula $=2^{\Delta \Delta C t}$. A cut-off copy number of 1.5 was used to define $p f m d r 1$ amplification. Reactions were repeated whenever the profile did not conform to exponential kinetics, or $\Delta \Delta \mathrm{Ct}$ spread was $>1.5$, or the Ct value was $>35$. To confirm amplification and resolve indeterminate results, samples passing these criteria but with an estimated copy number $>1.3$ were also re-tested once, the second result counting as final. For the three Cambodian sites where data have already been published, a conservative copy number cutoff of 1.7 was used to define amplification [20].

Pfmdr1 polymorphism was examined at codons 86 , $184,1034,1042$, and 1246 via PCR-restriction fragment length polymorphism (PCR-RFLP) using an established protocol [28].

Comparison of polymorphism results for samples successfully assessed by both PCR and WGS, based on whether pfmdr1 was categorized as amplified or not, were analysed by the kappa statistic. To calculate the overall proportion of samples with amplified $p f m d r 1$ at each site, the WGS-derived result was used where available; otherwise the corresponding PCR result was used (191 samples).

\section{Ethics}

The samples were tested under existing ethical approvals from the TRAC coordinating centre and individual sites [5]; additional ethical approval was obtained from the Ethics Committee of the Faculty of Tropical Medicine, Mahidol University, Bangkok, for laboratory work in Thailand. The TRAC study is registered with ClinicalTrials.gov (NCT01240603). 


\section{Results}

\section{Pfmdr1 amplification}

Overall, 998 samples had pfmdr1 copy number measured by PCR, WGS, or both. PCR-based assessment of $p f m d r 1$ copy number was successful in 332 samples tested in Thailand, adding to the 360 published results for three Cambodian sites (Table 1; Fig. 2a). Pfmdr1 copy number was also assessed in 807 samples for which WGS data were available (Fig. 2b). Comparison of results for the samples successfully assessed by both PCR and WGS, based on whether $p f m d r 1$ was categorized as amplified or not, indicated 93.2\% (467/501) agreement between methods $(\kappa=0.766,95 \%$ CI $0.691-0.841)$. To calculate the
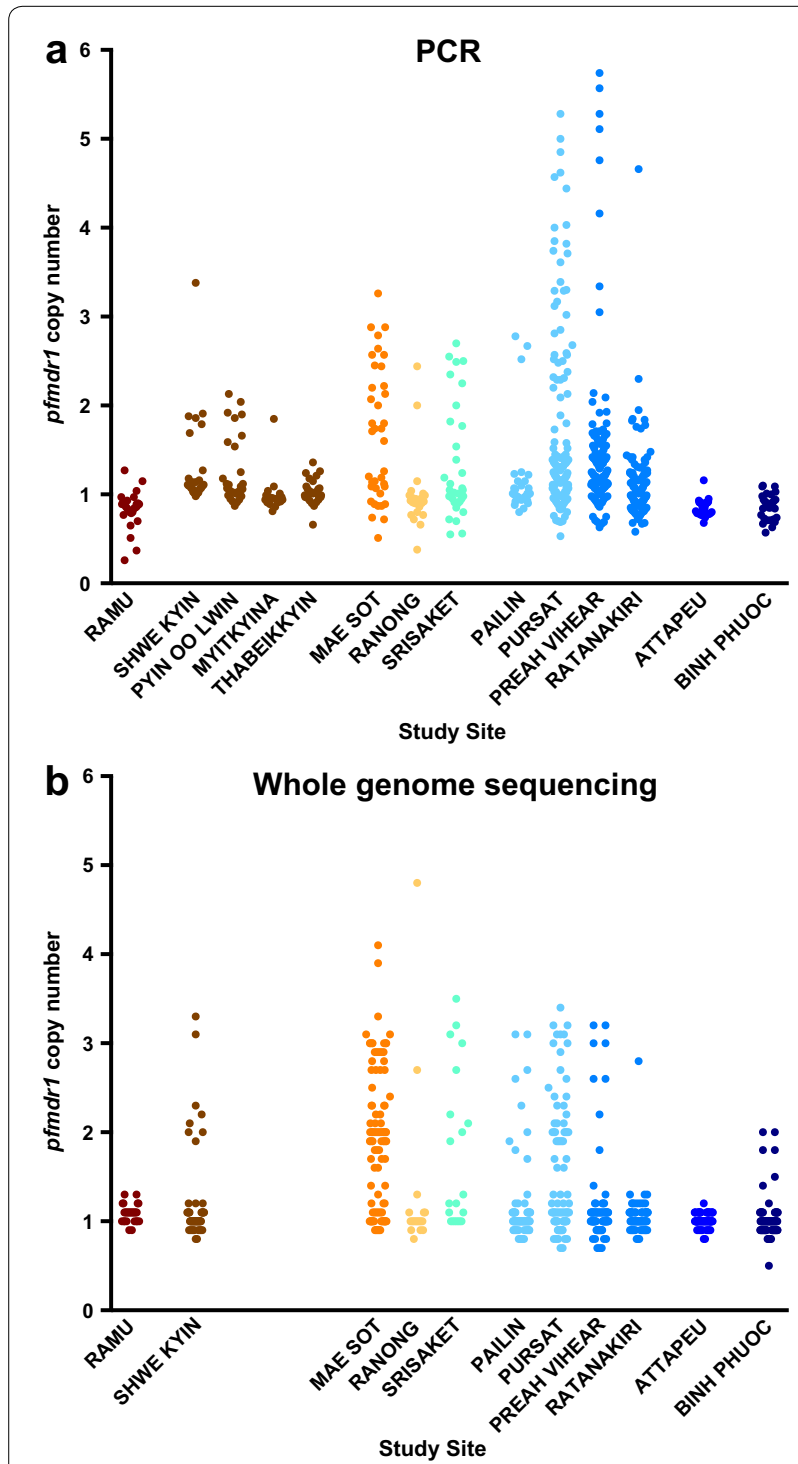

Fig. 2 Distribution of pfmdr1 copy number across sites measured by PCR (a) or Illumina sequencing ( $(\mathbf{b})$. The numbers of samples assessed at each site were not the same for the two methods (see Table 1) overall proportion of samples with amplified $p f m d r 1$ at each site, the WGS-derived result was used where available; otherwise the corresponding PCR result was used (191 samples). The proportions of samples with amplification according to site are shown graphically in Fig. 3 and in tabular format in Additional file 4.

Samples with amplified $p f m d r 1$ were found at all three sites in Thailand, being more prevalent in Mae Sot near the Myanmar border (59.8\%) and Srisaket (33.3\%) near the Cambodia border (Fig. 3). Amplification was also seen at lower levels in central Myanmar at Shwe Kyin (12.5\%) and Pyin Oo Lwin (28.0\%). In Cambodia, amplification was common in Pursat (40\%) but not at three other sites. The proportion of isolates with amplification was low or zero in Bangladesh, northern Myanmar, Laos, and Vietnam.

To investigate how the geographical distribution of $p f m d r 1$ amplification relates to the genetic structure of the parasite population, samples with WGS data were plotted on a neighbour-joining tree, which groups samples according to genome-wide genetic similarity as previously described [19] (Fig. 4). In the 'western SEA' compartment [19] (Mae Sot and Ranong at the ThailandMyanmar border and Shwe Kyin in central Myanmar), pfmdr1 amplification was evenly dispersed, while in the more structured populations of western and northern Cambodia (Pailin, Pursat, and Preah Vihear) and eastern Thailand (Srisaket), pfmdr1 amplification tended to cluster within particular branches.

\section{Pfmdr1 polymorphism}

The polymorphic positions N86Y, Y184F, S1034C, N1042D, and D1246Y were assessed by both PCR-RFLP and Illumina methods. Overall, agreement was $97.4 \%$ ( $\mathrm{k}=0.825,95 \%$ CI $0.765-0.885$ ), with most disagreements consisting of heterozygous calls by WGS that were assigned a homozygous genotype by PCR-RFLP. Samples that yielded a heterozygous genotype by either method, or for which the two methods produced discordant homozygous genotypes (six calls), were considered as having mixed alleles at that position, and apportioned equally between the two alleles for the purposes of calculating allele frequency. Summary results for all five positions are reported in tabular format in Additional file 5.

The N86Y mutation was found in 19\% of samples in Bangladesh and in fewer than $5 \%$ of samples at all other sites (Fig. 5a). The N86F mutation [29] was also observed in three mixed samples in Bangladesh. The Y184F mutation was found in more than $10 \%$ of parasites at most sites, being particularly common (85-90\% of samples) in western Cambodia (Pailin and Pursat) and eastern Thailand (Srisaket) (Fig. 5b). The N1042D mutation was rare (ten samples including four mixed alleles), while the S1034C and D1246Y mutations were entirely absent from the sample set. 


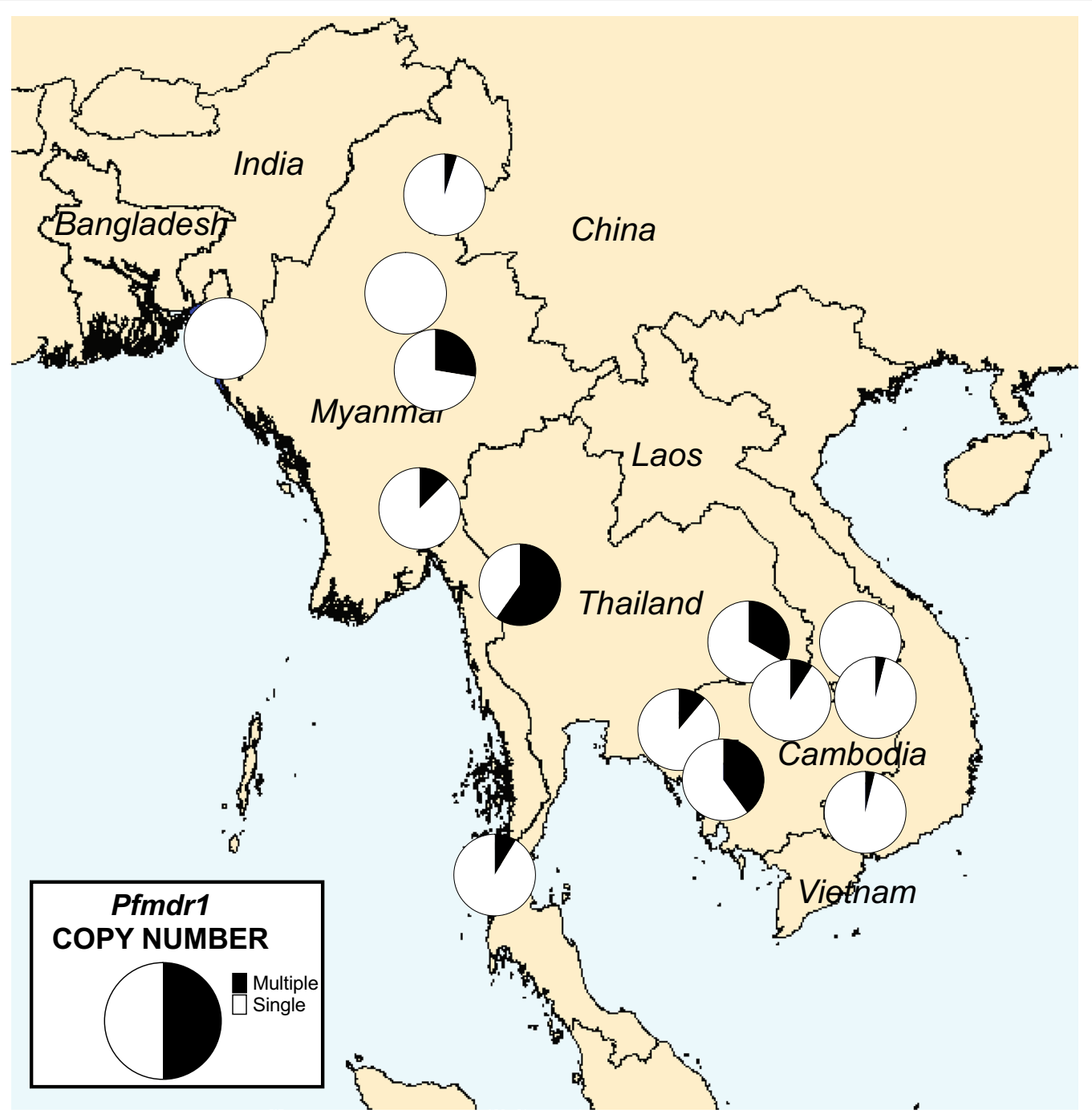

Fig. 3 Proportion of isolates with pfmdrl amplification at each site

\section{Other pfmdr1 polymorphisms}

Analysis of WGS data also identified 11 additional pfmdr1 SNPs distinct from the five SNPs commonly assessed (Additional file 6). While these were generally found at low frequencies, in six cases the derived allele had a frequency of more than $10 \%$ in at least one site (Additional file 7). The F1226Y mutation was found in 54\% of samples in Mae Sot. The A784L mutation was found in $>20 \%$ of samples in Pailin, Cambodia and Shwe Kyin, Myanmar.

\section{Pfcrt polymorphism}

The great majority (98.2\%) of samples across the study contained parasites carrying the key chloroquine resistance mutation K76T; only 14 samples carrying the wildtype allele were distributed across Bangladesh, Laos, northeastern Cambodia, and Vietnam (Fig. 6). At residues 72-76, the CVIET haplotype was predominant (95\% or more) in Bangladesh, Myanmar, and Thailand, while the CVIDT haplotype was also common in northern and northeastern Cambodia, Laos, and Vietnam (33-38\%). No sample carried the SVMNT haplotype associated with high levels of amodiaquine resistance [30].

\section{Discussion}

This large survey of the molecular markers $p f m d r 1$ and pfcrt offers insights into patterns of anti-malarial partner drug susceptibility across mainland SEA, which reflect the history of anti-malarial use and may guide future therapeutic studies at each location.

Pfmdr1 amplification is associated with reduced efficacy of both artemether-lumefantrine, globally the 


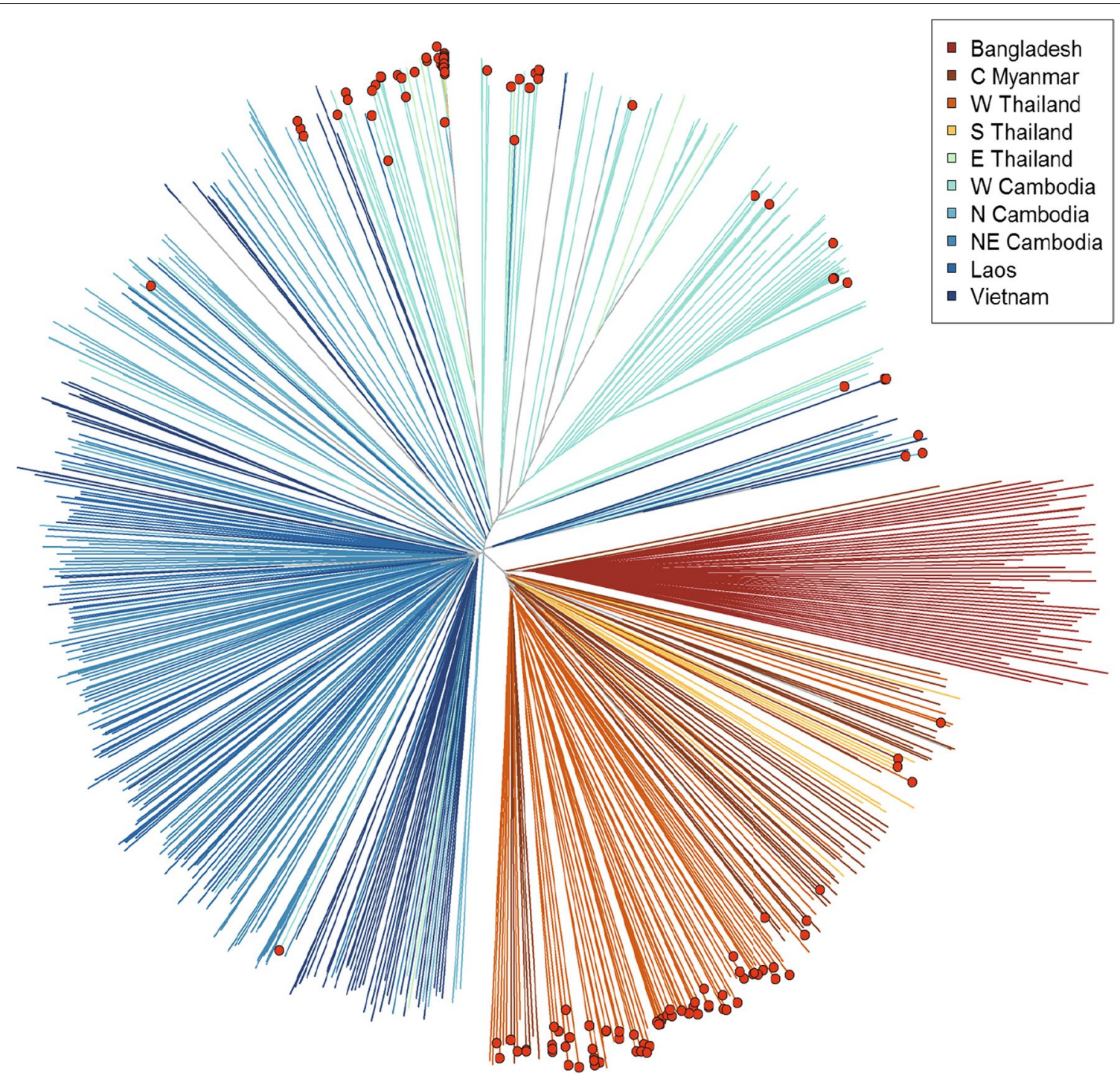

Fig. 4 Neighbour-joining tree showing population structure across 11 sites studied by Illumina sequencing. Branches with coloured tip symbols indicate that the samples have pfmdr 1 amplification

most widely used ACT [14], and artesunate-mefloquine. Pfmdr1 copy number can be assessed by a range of methods [31]. Most surveys of field isolates have used quantitative PCR focused on the individual gene [32], while genome-wide studies of specific laboratory isolates have used microarray-based or next-generation approaches [33-35]. Here, a comparison of PCR-based and next-generation sequencing data in approximately 500 samples showed good agreement between the two approaches.

Artemether-lumefantrine is the currently recommended first-line treatment in Bangladesh, Myanmar, and Laos. The presence of substantial numbers of $P$. falciparum isolates with $p f m d r 1$ amplification in Shwe Kyin (Bago Province) and Pyin Oo Lwin (Mandalay Province), combined with results from a smaller number of samples obtained from nearby sites [36] indicates that isolates with amplified $p f m d r 1$ are common in central Myanmar. High levels of $p f m d r 1$ amplification are also present along the Thailand-Myanmar border, where they have persisted for over two decades [15, 37], although the efficacy of artesunate-mefloquine has dropped to unacceptable levels only within the last 5 years, following the emergence of artemisinin resistance $[4,7,38]$.

Isolates with amplified pfmdr1 were also seen in Ranong (southern Thailand-Myanmar border), consistent with previous data [39-41]. Analysis of genomewide genetic similarity shows that parasites from the Thailand-Myanmar border and central Myanmar (Shwe 


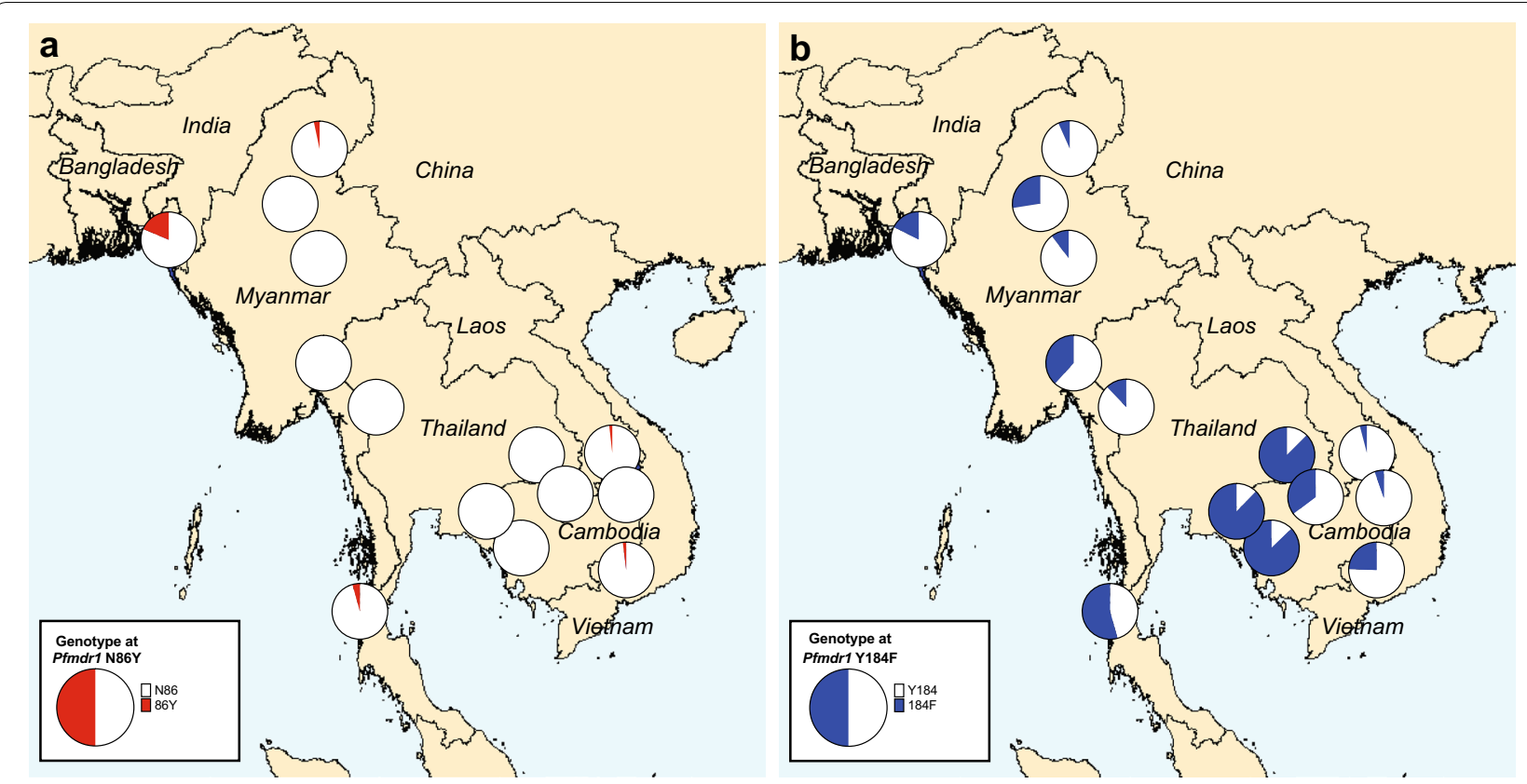

Fig. 5 Mutant allele frequency per site for the pfmdr1 SNPs N86Y (a) and Y184F (b)

Kyin) are related; it is worth noting that most patients enrolled in Mae Sot travelled from Myanmar, with the catchment area extending as far as $40 \mathrm{~km}$ inside the border [7]. Samples with amplified pfmdr1 are evenly distributed within this parasite population compartment, rather than associated with any specific sub-population. At the two sites in northern Myanmar (Thabeikkyin and Myitkyina), pfmdr1 amplification appears to be at low prevalence, consistent with previous data from nearby border areas [42]. However, there is clearly a need for close observation of the efficacy of artemether-lumefantrine in Myanmar given that reduced parasite clearance rates after ACT have been observed in border areas [43, 44] and K13-propeller mutations that confer artemisinin resistance are widespread [45].

Pfmdr1 amplification was also present at Srisaket in eastern Thailand near the Cambodian border, consistent with continued mefloquine usage, following its adoption as first-line therapy in 2007. Given the widespread nature of samples with K13-propeller mutations and slow clearance following artesunate treatment [5], there is concern that artesunate-mefloquine efficacy will be compromised across a wider area of Thailand in the near future. These data match previously published data from a distinct area of the Thailand-Cambodia border $[39,46]$.

Pfmdr1 amplification was found at Pailin and Pursat in western Cambodia, although for Pailin this was at lower levels compared to 2004 [17] when artesunate-mefloquine had unacceptably low efficacy [6]. This likely reflects the 2008-2010 change of policy, leading to the adoption of dihydroartemisinin-piperaquine as the frontline ACT across Cambodia. Since the TRAC study, there has been further reduction in the prevalence of parasites with increased $p f m d r 1$ copy number in western and northern Cambodian [10, 47, 48]. In Cambodia, pfmdr1 amplification tended to be found within specific sub-populations in these locations, probably as a result of pronounced population structure caused by the expansion of artemisinin-resistant founder populations [49].

Laos has been using artemether-lumefantrine since 2001, but there was no evidence of $p f m d r 1$ amplification. This is likely to reflect the introduction of artemetherlumefantrine as national policy without a prior period of mefloquine monotherapy, with maintained artemisinin sensitivity up to the time of this study [5], and is consistent with the documented high efficacy of both artemether-lumefantrine and artesunate-mefloquine [50]. Vietnam has a distinct history of antimalarial use, with dihydroartemisinin-piperaquine deployed as first-line therapy in 2005 (taking over from artesunate plus mefloquine). Consistent with the longstanding absence of mefloquine or lumefantrine from antimalarial therapy, few parasites from Vietnam showed $p f m d r 1$ amplification.

SNPs in pfmdr1 may provide additional relevant information for guiding anti-malarial policy and planning further studies. The N86Y polymorphism is of relevance to aminoquinoline sensitivity (see below). Y184F 


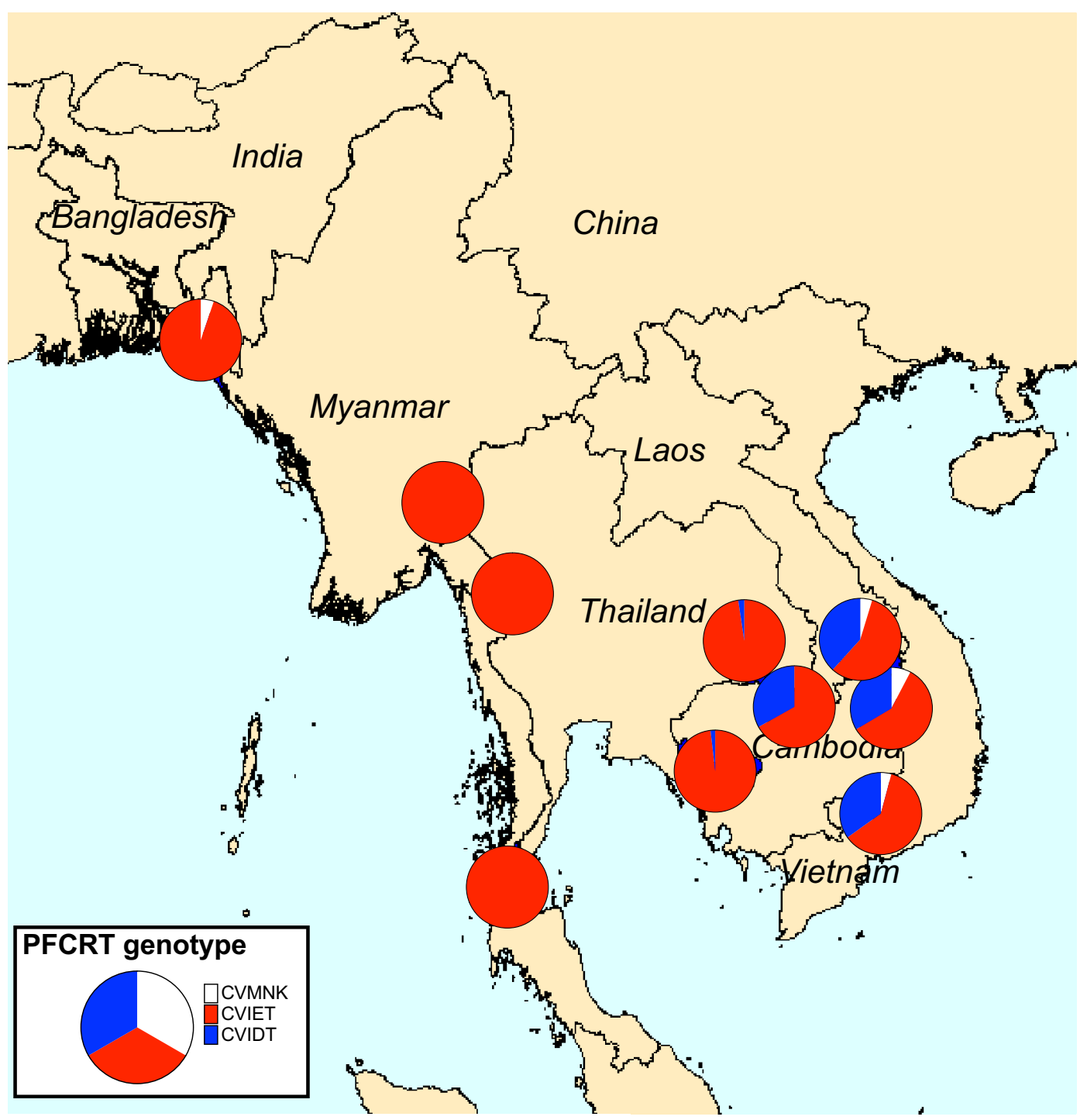

Fig. 6 Proportion of isolates with each of three pfcrt haplotypes encoding amino acids 72-76 (11 sites). The pfcrt SVMNT haplotype was not found in any sample

polymorphism was most common, especially in western Cambodia and eastern Thailand, and WGS data also revealed other $p f m d r 1$ mutations (e.g., the F1226Y mutation prevalent in Mae Sot). Both of these mutations have been associated with decreased in vitro susceptibility to mefloquine [51, 52], although most studies have not found Y184F to be significantly associated with changes in drug responses [53, 54]. Further studies are needed to determine whether these mutations are being naturally selected for drug resistance or other phenotypes.

The pfcrt K76T mutation was at or near fixation in all study sites, with the CVIET haplotype dominant in western SEA and the CVIDT haplotype found in approximately one-third of isolates from northern and northeastern Cambodia [55], southern Laos, and southern Vietnam. The SVMNT pfcrt haplotype associated with high-grade amodiaquine resistance was not found in any location, in contrast to a study in 2003 in northern Laos [56] and other areas of SEA [57]. The pfmdr1 N86Y mutation, which plays a role in amodiaquine resistance $[13,30]$, has all but disappeared from the region following abandonment of chloroquine and the use of mefloquine in the period leading up to this study, and in line with other observations [58]. For these reasons, amodiaquine may show some useful efficacy in SEA. This is also consistent with the acceptable clinical efficacy of artesunate-amodiaquine in some [59,60], but not all [61], parts of SEA within the last decade. Furthermore, the inverse 
correlation between susceptibility to 4-aminoquinolines and mefloquine $[62,63]$ offers the potential to combine two partners with opposing resistance mechanisms within a novel 'triple' ACT, artemether-lumefantrine plus amodiaquine [64]; this is currently under investigation in the follow-up study to TRAC (ClinicalTrials.gov NCT02453308).

\section{Conclusions}

In summary, these data offer new insights into partner drug resistance patterns across a large area of SEA. The finding that $p f m d r 1$ amplification, associated with mefloquine and lumefantrine resistance, extends into central Myanmar is concerning and highlights the need for close observation of the efficacy of artemether-lumefantrine in Myanmar. In contrast, the reduction in $p f m d r 1$ amplification in western Cambodia and lower areas of the Greater Mekong Subregion supports the use (or re-use) of artesunate-mefloquine in areas where dihydroartemisinin-piperaquine efficacy is unacceptable. Finally, SNP patterns in $p f m d r 1$ and $p f c r t$ suggest that amodiaquine may improve treatment efficacy if it can be practically incorporated into anti-malarial regimens.

\section{Additional files}

Additional file 1. Reference positions used to estimate pfmdr 1 copy number from WGS coverage data.

Additional file 2. Screenshot of the MalariaGEN P. falciparum Community Project web application (http://www.malariagen.net/apps/pf/4.0/). Location of six reference positions located within gene PF3D7_ 1455600 (ferlin, putative).

Additional file 3. Histogram of estimated pfmdr1 copy number in the present dataset, estimated from WGS reads coverage. Each bin represents an estimate value interval of 0.1. A kernel density estimate of these data is overlaid (dark blue curve) to show the location of peaks, derived from the density() function in the R stats package, using a Gaussian kernel with standard deviation 0.15 . There are clear peaks in sample numbers at integer values of $p f m d r 1$ copy number $\left(N_{\text {est }}=\{1,2,3\}\right)$, and troughs at intermediate positions, consistent with the expected distribution of pfmdr 1 copy number values.

Additional file 4. Proportion of samples with amplified pfmdr1, based on a combination of PCR and Illumina-based sequencing.

Additional file 5. Numbers of isolates with pure wild-type, mutant or mixed alleles at five well-described polymorphic sites in pfmdr 1 (combination of PCR-RFLP and Illumina methods). *Three samples were mixed N86/86F infections.

Additional file 6. Numbers of isolates with pure wild-type, mutant, or mixed alleles at eleven additional polymorphic sites in pfmdr 1 (Illumina method only).

Additional file 7. Proportions of mutant pfmdr 1 alleles at six other polymorphic positions (11 study sites).

\section{Authors' contributions}

All authors contributed to study design and data analysis. KS, PL and MI undertook laboratory work. KS, OM, RMF, CJW, and MI drafted the manuscript. All authors read and approved the final manuscript.

\section{Author details}

${ }^{1}$ Department of Molecular Tropical Medicine and Genetics, Faculty of Tropical Medicine, Mahidol University, Bangkok, Thailand. ${ }^{2}$ Mahidol Oxford Tropical Medicine Research Unit, Bangkok, Thailand. ${ }^{3}$ Wellcome Trust Sanger Institute, Hinxton, UK. ${ }^{4}$ Medical Research Council (MRC) Centre for Genomics and Global Health, University of Oxford, Oxford, UK. ${ }^{5}$ Laboratory of Malaria and Vector Research, National Institute of Allergy and Infectious Diseases, National Institutes of Health, Rockville, MD 20852, USA. ${ }^{6}$ Centre for Tropical Medicine \& Global Health, Nuffield Department of Medicine, University of Oxford, Oxford, UK.

\section{Acknowledgements}

We thank the healthcare workers involved in sample collection across all sites.

Additional Tracking Resistance to Artemisinin Collaboration authors (TRAC Group Authorship):

Oxford University Clinical Research Unit, Hospital for Tropical Diseases, Ho

Chi Minh City, Vietnam

Tinh Hien Tran, Nguyen Thanh Thuy

Department of Medical Research, Lower Myanmar, Myanmar Ye Htut

Department of Medical Research, Upper Myanmar, Myanmar Khin Lin

Defence Services Medical Research Centre, Naypyitaw, Myanmar Kyaw Myo Tun, Tin M. Hlaing

Laos-Oxford-Mahosot Hospital-Wellcome Trust Research Unit, Mahosot Hospital, Vientiane, Laos

Mayfong Mayxay, Paul N. Newton

The Malaria Research Group and Dev Care Foundation, Dhaka, Bangladesh Md. Abul Faiz

Shoklo Malaria Research Unit, Mae Sot, Thailand

Aung Pyae Phyo, François Nosten

Mahidol-Oxford Tropical Medicine Research Unit, Bangkok, Thailand Elizabeth A. Ashley, Mehul Dhorda, Sasithon Pukrittayakamee, Frank Smithuis, Nicholas P. J. Day, Arjen M. Dondorp, Nicholas J. White.

\section{Competing interests}

The authors declare that they have no competing interests.

\section{Availability of data and material}

The datasets during and/or analysed during the current study available from the corresponding author on reasonable request.

\section{Ethics approval and consent to participate}

The samples were tested under existing ethical approvals from the TRAC coordinating centre and individual sites [5]; additional ethical approval was obtained from the Ethics Committee of the Faculty of Tropical Medicine, Mahidol University, Bangkok, for laboratory work in Thailand. The TRAC study is registered with ClinicalTrials.gov (NCT01240603).

\section{Funding}

PCR work in Thailand was funded by the Royal Golden Jubilee Ph.D. Programme, the Thailand Research Fund, and Mahidol University. The MahidolOxford Tropical Medicine Research Programme is funded by the Wellcome Trust of Great Britain. Whole-genome sequencing for this study was funded by the Wellcome Trust through core funding of the Wellcome Trust Sanger Institute (098051). The Wellcome Trust also supports the Wellcome Trust Centre for Human Genetics (090532/Z/09/Z) and the Resource Centre for Genomic Epidemiology of Malaria (090770/Z/09/Z). The Centre for Genomics and Global Health is supported by the UK Medical Research Council (G0600718, MR/M006212/1). This work was funded in part by the Bill and Melinda Gates Foundation (OPP1040463). The fieldwork to obtain samples was supported by grants from the UK Department for International Development, the Worldwide Anti-malarial Resistance Network (WWARN), and the Intramural Research Program of the National Institute of Allergy and Infectious Diseases, US National Institutes of Health. The views expressed and information contained in this article are not necessarily those of or endorsed by the UK Department for International Development or by the other funders, which can accept no responsibility for such views or for any reliance placed on them. 
Received: 6 August 2016 Accepted: 31 October 2016 Published online: 08 November 2016

\section{References}

1. Noedl H, Socheat D, Satimai W. Artemisinin-resistant malaria in Asia. N Engl J Med. 2009;361:540-1.

2. Dondorp AM, Nosten F, Yi P, Das D, Phyo AP, Tarning J, et al. Artemisinin resistance in Plasmodium falciparum malaria. N Engl J Med. 2009:361:455-67.

3. Amaratunga C, Sreng S, Suon S, Phelps ES, Stepniewska K, Lim P, et al. Artemisinin-resistant Plasmodium falciparum in Pursat province, western Cambodia: a parasite clearance rate study. Lancet Infect Dis. 2012;12:851-8

4. Phyo AP, Nkhoma S, Stepniewska K, Ashley EA, Nair S, McGready R, et al. Emergence of artemisinin-resistant malaria on the western border of Thailand: a longitudinal study. Lancet. 2012;379:1960-6.

5. Ashley EA, Dhorda M, Fairhurst RM, Amaratunga C, Lim P, Suon S, et al. Spread of artemisinin resistance in Plasmodium falciparum malaria. N Engl J Med. 2014;371:411-23.

6. Denis MB, Tsuyuoka R, Poravuth Y, Narann TS, Seila S, Lim C, et al. Surveillance of the efficacy of artesunate and mefloquine combination for the treatment of uncomplicated falciparum malaria in Cambodia. Trop Med Int Health. 2006;11:1360-6.

7. Carrara VI, Lwin KM, Phyo AP, Ashley E, Wiladphaingern J, Sriprawat K, et al. Malaria burden and artemisinin resistance in the mobile and migrant population on the Thai-Myanmar border, 1999-2011: an observational study. PLoS Med. 2013;10:e1001398.

8. Leang R, Barrette A, Bouth DM, Menard D, Abdur R, Duong S, et al. Efficacy of dihydroartemisinin-piperaquine for treatment of uncomplicated Plasmodium falciparum and Plasmodium vivax in Cambodia, 2008 to 2010 Antimicrob Agents Chemother. 2013:57:818-26.

9. Saunders DL, Vanachayangkul P, Lon C. Dihydroartemisinin-piperaquine failure in Cambodia. N Engl J Med. 2014;371:484-5.

10. Amaratunga C, Lim P, Suon S, Sreng S, Mao S, Sopha C, et al. Dihydroartemisinin-piperaquine resistance in Plasmodium falciparum malaria in Cambodia: a multisite prospective cohort study. Lancet Infect Dis. 2016;16:357-65.

11. Laufer MK, Djimde AA, Plowe CV. Monitoring and deterring drugresistant malaria in the era of combination therapy. Am J Trop Med Hyg 2007;77:160-9.

12. Petersen I, Gabryszewski SJ, Johnston GL, Dhingra SK, Ecker A, Lewis $\mathrm{RE}$, et al. Balancing drug resistance and growth rates via compensatory mutations in the Plasmodium falciparum chloroquine resistance transporter. Mol Microbiol. 2015;97:381-95

13. Veiga MI, Dhingra SK, Henrich PP, Straimer J, Gnadig N, Uhlemann AC, et al. Globally prevalent PFMDR1 mutations modulate Plasmodium falciparum susceptibility to artemisinin-based combination therapies. Nat Commun. 2016;7:11553.

14. Venkatesan M, Gadalla NB, Stepniewska K, Dahal P, Nsanzabana C, Moriera $\mathrm{C}$, et al. Polymorphisms in Plasmodium falciparum chloroquine resistance transporter and multidrug resistance 1 genes: parasite risk factors that affect treatment outcomes for $P$. falciparum malaria after artemether-lumefantrine and artesunate-amodiaquine. Am J Trop Med Hyg. 2014;91:833-43.

15. Price RN, Uhlemann AC, Brockman A, McGready R, Ashley E, Phaipun $L$, et al. Mefloquine resistance in Plasmodium falciparum and increased pfmdr1 gene copy number. Lancet. 2004:364:438-47.

16. Sidhu AB, Uhlemann AC, Valderramos SG, Valderramos JC, Krishna S, Fidock DA. Decreasing pfmdr 1 copy number in Plasmodium falciparum malaria heightens susceptibility to mefloquine, lumefantrine, halofantrine, quinine, and artemisinin. J Infect Dis. 2006;194:528-35.

17. Alker AP, Lim P, Sem R, Shah NK, Yi P, Bouth DM, et al. Pfmdr 1 and in vivo resistance to artesunate-mefloquine in falciparum malaria on the Cambodian-Thai border. Am J Trop Med Hyg. 2007;76:641-7.

18. Lim P, Alker AP, Khim N, Shah NK, Incardona S, Doung S, et al. Pfmdr copy number and arteminisin derivatives combination therapy failure in falciparum malaria in Cambodia. Malar J. 2009;8:11

19. Miotto $O$, Amato R, Ashley EA, Maclnnis B, Almagro-Garcia J, Amaratunga $C$, et al. Genetic architecture of artemisinin-resistant Plasmodium falciparum. Nat Genet. 2015:47:226-34.
20. Lim P, Dek D, Try V, Eastman RT, Chy S, Sreng S, et al. Ex vivo susceptibility of Plasmodium falciparum to antimalarial drugs in western, northern and eastern Cambodia, 2011-2012: association with molecular markers. Antimicrob Agents Chemother. 2013;57:5277-83.

21. Venkatesan M, Amaratunga C, Campino S, Auburn S, Koch O, Lim P, et al. Using CF11 cellulose columns to inexpensively and effectively remove human DNA from Plasmodium falciparum-infected whole blood samples. Malar J. 2012;11:41

22. Manske M, Miotto O, Campino S, Auburn S, Almagro-Garcia J, Maslen G, et al. Analysis of Plasmodium falciparum diversity in natural infections by deep sequencing. Nature. 2012:487:375-9.

23. MalariaGEN Plasmodium falciparum Community Project. Genomic epidemiology of artemisinin resistant malaria. Elife. 2016;5. doi:10.7554/ eLife.08714.

24. http://www.malariagen.net/projects/parasite/pf and http://www.malariagen.net/apps/pf/.

25. http://www.htslib.org/.

26. Imwong M, Dondorp AM, Nosten F, Yi P, Mungthin M, Hanchana S, et al. Exploring the contribution of candidate genes to artemisinin resistance in Plasmodium falciparum. Antimicrob Agents Chemother. 2010;54:2886-92.

27. Preechapornkul $\mathrm{P}$, Imwong $M$, Chotivanich $K$, Pongtavornpinyo $W$ Dondorp AM, Day NP, et al. Plasmodium falciparum pfmdr1 amplification, mefloquine resistance, and parasite fitness. Antimicrob Agents Chemother. 2009;53:1509-15.

28. Duraisingh MT, Jones P, Sambou I, von Seidlein L, Pinder M, Warhurst DC. The tyrosine-86 allele of the pfmdr1 gene of Plasmodium falciparum is associated with increased sensitivity to the anti-malarials mefloquine and artemisinin. Mol Biochem Parasitol. 2000;108:13-23.

29. Beshir K, Sutherland CJ, Merinopoulos I, Durrani N, Leslie T, Rowland $M$, et al. Amodiaquine resistance in Plasmodium falciparum malaria in Afghanistan is associated with the pfcrt SVMNT allele at codons 72 to 76. Antimicrob Agents Chemother. 2010;54:3714-6.

30. Sa JM, Twu O, Hayton K, Reyes S, Fay MP, Ringwald P, et al. Geographic patterns of Plasmodium falciparum drug resistance distinguished by differential responses to amodiaquine and chloroquine. Proc Natl Acad Sci USA. 2009:106:18883-9.

31. Anderson TJ, Patel J, Ferdig MT. Gene copy number and malaria biology. Trends Parasitol. 2009:25:336-43.

32. Price R, Robinson G, Brockman A, Cowman A, Krishna S. Assessment of pfmdr1 gene copy number by tandem competitive polymerase chain reaction. Mol Biochem Parasitol. 1997;85:161-9.

33. Ribacke U, Mok BW, Wirta V, Normark J, Lundeberg J, Kironde F, et al. Genome wide gene amplifications and deletions in Plasmodium falciparum. Mol Biochem Parasitol. 2007;155:33-44.

34. Kidgell C, Volkman SK, Daily J, Borevitz JO, Plouffe D, Zhou Y, et al. A systematic map of genetic variation in Plasmodium falciparum. PLoS Pathog. 2006;2:e57.

35. Hunt P, Martinelli A, Modrzynska K, Borges S, Creasey A, Rodrigues L, et al. Experimental evolution, genetic analysis and genome re-sequencing reveal the mutation conferring artemisinin resistance in an isogenic lineage of malaria parasites. BMC Genom. 2010;11:499.

36. Win AA, Imwong M, Kyaw MP, Woodrow CJ, Chotivanich K, Hanboonkunupakarn B, et al. K13 mutations and pfmdr1 copy number variation in Plasmodium falciparum malaria in Myanmar. Malar J. 2016;15:110.

37. Carrara VI, Zwang J, Ashley EA, Price RN, Stepniewska K, Barends M, et al. Changes in the treatment responses to artesunate-mefloquine on the northwestern border of Thailand during 13 years of continuous deployment. PLoS ONE. 2009;4:e4551.

38. Pyae Phyo A, Ashley EA, Anderson TJ, Bozdech Z, Carrara VI, Sriprawat K, et al. Declining efficacy of artemisinin combination therapy against $P$. falciparum malaria on the Thai-Myanmar border (2003-2013): the role of parasite genetic factors. Clin Infect Dis. 2016:63:784-91.

39. Mungthin M, Khositnithikul R, Sitthichot N, Suwandittakul N, Wattanaveeradej $\mathrm{V}$, Ward SA, et al. Association between the pfmdr 1 gene and in vitro artemether and lumefantrine sensitivity in Thai isolates of Plasmodium falciparum. Am J Trop Med Hyg. 2010;83:1005-9.

40. Muhamad P, Chaijaroenkul W, Phompradit P, Rueangweerayut R, Tippawangkosol P, Na-Bangchang K. Polymorphic patterns of pfert and pfmdr 1 in Plasmodium falciparum isolates along the Thai-Myanmar border. Asian Pac J Trop Biomed. 2013:3:931-5. 
41. Mungthin M, Intanakom S, Suwandittakul N, Suida P, Amsakul S, Sitthichot N, et al. Distribution of pfmdr1 polymorphisms in Plasmodium falciparum isolated from Southern Thailand. Malar J. 2014;13:117.

42. Brown T, Smith LS, Oo EK, Shawng K, Lee TJ, Sullivan D, et al. Molecular surveillance for drug-resistant Plasmodium falciparum in clinical and subclinical populations from three border regions of Burma/Myanmar: cross-sectional data and a systematic review of resistance studies. Malar J. 2012;11:333.

43. Takala-Harrison S, Jacob CG, Arze C, Cummings MP, Silva JC, Dondorp $\mathrm{AM}$, et al. Independent emergence of artemisinin resistance mutations among Plasmodium falciparum in Southeast Asia. J Infect Dis. 2015;211:670-9.

44. Wang Z, Wang Y, Cabrera M, Zhang Y, Gupta B, Wu Y, et al. Artemisinin resistance at the China-Myanmar border and association with mutations in the K13 propeller gene. Antimicrob Agents Chemother. 2015;59:6952-9.

45. Tun KM, Imwong M, Lwin KM, Win AA, Hlaing TM, Hlaing T, et al. Spread of artemisinin-resistant Plasmodium falciparum in Myanmar: a cross-sectional survey of the K13 molecular marker. Lancet Infect Dis. 2015:15:415-21.

46. Mungthin M, Suwandittakul N, Chaijaroenkul W, Rungsrihirunrat K, Harnyuttanakorn P, Seugorn A, et al. The patterns of mutation and amplification of Plasmodium falciparum pfcrt and pfmdr1 genes in Thailand during the year 1988 to 2003. Parasitol Res. 2010;107:539-45.

47. Lim P, Dek D, Try V, Sreng S, Suon S, Fairhurst RM. Decreasing pfmdr1 copy number suggests that Plasmodium falciparum in Western Cambodia is regaining in vitro susceptibility to mefloquine. Antimicrob Agents Chemother. 2015;59:2934-7.

48. Chaorattanakawee S, Saunders DL, Sea D, Chanarat N, Yingyuen K, Sundrakes S, et al. Ex vivo drug susceptibility testing and molecular profiling of clinical Plasmodium falciparum isolates from Cambodia from 2008 to 2013 suggest emerging piperaquine resistance. Antimicrob Agents Chemother. 2015;59:4631-43.

49. Miotto O, Almagro-Garcia J, Manske M, Macinnis B, Campino S, Rockett KA, et al. Multiple populations of artemisinin-resistant Plasmodium falciparum in Cambodia. Nat Genet. 2013;45:648-55.

50. Mayxay M, Keomany S, Khanthavong M, Souvannasing P, Stepniewska K, Khomthilath T, et al. A phase III, randomized, non-inferiority trial to assess the efficacy and safety of dihydroartemisinin-piperaquine in comparison with artesunate-mefloquine in patients with uncomplicated Plasmodium falciparum malaria in southern Laos. Am J Trop Med Hyg. 2010;83:1221-9.

51. Khim N, Bouchier C, Ekala MT, Incardona S, Lim P, Legrand E, et al. Countrywide survey shows very high prevalence of Plasmodium falciparum multilocus resistance genotypes in Cambodia. Antimicrob Agents Chemother. 2005:49:3147-52.

52. Veiga MI, Ferreira PE, Jornhagen L, Malmberg M, Kone A, Schmidt BA, et al. Novel polymorphisms in Plasmodium falciparum ABC transporter genes are associated with major ACT antimalarial drug resistance. PLoS ONE. 2011:6:e20212.
53. Woodrow CJ, Krishna S. Antimalarial drugs: recent advances in molecular determinants of resistance and their clinical significance. Cell Mol Life Sci. 2006;63:1586-96.

54. Wurtz N, Fall B, Pascual A, Fall M, Baret E, Camara C, et al. Role of Pfmdr1 in in vitro Plasmodium falciparum susceptibility to chloroquine, quinine, monodesethylamodiaquine, mefloquine, lumefantrine, and dihydroartemisinin. Antimicrob Agents Chemother. 2014;58:7032-40.

55. Durrand V, Berry A, Sem R, Glaziou P, Beaudou J, Fandeur T. Variations in the sequence and expression of the Plasmodium falciparum chloroquine resistance transporter (Pfcrt) and their relationship to chloroquine resistance in vitro. Mol Biochem Parasitol. 2004;136:273-85.

56. Dittrich S, Alifrangis M, Stohrer JM, Thongpaseuth V, Vanisaveth V, Phetsouvanh $\mathrm{R}$, et al. Falciparum malaria in the north of Laos: the occurrence and implications of the Plasmodium falciparum chloroquine resistance transporter (pfcrt) gene haplotype SVMNT. Trop Med Int Health. 2005; 10:1267-70.

57. Mehlotra RK, Fujioka H, Roepe PD, Janneh O, Ursos LM, Jacobs-Lorena V, et al. Evolution of a unique Plasmodium falciparum chloroquine-resistance phenotype in association with pfcrt polymorphism in Papua New Guinea and South America. Proc Natl Acad Sci USA. 2001:98:12689-94.

58. Malmberg M, Ngasala B, Ferreira PE, Larsson E, Jovel I, Hjalmarsson A, et al. Temporal trends of molecular markers associated with artemetherlumefantrine tolerance/resistance in Bagamoyo district, Tanzania. Malar J. 2013;12:103.

59. Thanh NX, Trung TN, Phong NC, Quang HH, Dai B, Shanks GD, et al. The efficacy and tolerability of artemisinin-piperaquine (Artequick $(R))$ versus artesunate-amodiaquine (Coarsucam) for the treatment of uncomplicated Plasmodium falciparum malaria in south-central Vietnam. Malar J. 2012;11:217.

60. Thanh NX, Trung TN, Phong NC, Thien NX, Dai B, Shanks GD, et al. Open label randomized comparison of dihydroartemisinin-piperaquine and artesunate-amodiaquine for the treatment of uncomplicated Plasmodium falciparum malaria in central Vietnam. Trop Med Int Health. 2009;14:504-11.

61. Smithuis F, Kyaw MK, Phe O, Win T, Aung PP, Oo AP, et al. Effectiveness of five artemisinin combination regimens with or without primaquine in uncomplicated falciparum malaria: an open-label randomised trial. Lancet Infect Dis. 2010;10:673-81.

62. Wernsdorfer WH, Landgraf B, Wiedermann G, Kollaritsch H. Inverse correlation of sensitivity in vitro of Plasmodium falciparum to chloroquine and mefloquine in Ghana. Trans R Soc Trop Med Hyg. 1994;88:443-4.

63. Sidhu AB, Verdier-Pinard D, Fidock DA. Chloroquine resistance in Plasmodium falciparum malaria parasites conferred by pfcrt mutations. Science. 2002:298:210-3.

64. Woodrow CJ, White NJ. The clinical impact of artemisinin resistance in Southeast Asia and the potential for future spread. FEMS Microbiol Rev. 2016 (Epub ahead of print)

\section{Submit your next manuscript to BioMed Central and we will help you at every step:}

- We accept pre-submission inquiries

- Our selector tool helps you to find the most relevant journal

- We provide round the clock customer support

- Convenient online submission

- Thorough peer review

- Inclusion in PubMed and all major indexing services

- Maximum visibility for your research

Submit your manuscript at www.biomedcentral.com/submit
BioMed Central 